\title{
Eine quantitative Methode zur gleichzeitigen Bestimmung von Thiamin und Thiaminpyrophosphat in Lebensmitteln und Ausscheidungen
}

\author{
Von LIESEL WILDEMANN \\ Max-Planck-Institut für Ernäbrungsphysiologie Dortmund
}

(Eingegangen am 30. Mai 1969)

Es wird eine einfache, spezifische und schnelle Methode für die Bestimmung von Thiamin und Thiaminpyrophosphat beschrieben. Eine Hydrolyse der Phosphatgruppen ist nicht erforderlich. Nach Enteiweißung wird die Probe über eine Sephadex-G-25-Säule gegeben und mit dem Thiochromtest bestimmt. Die beste Reproduzierbarkeit wurde im Bereich 0,5-3 $\mu \mathrm{g}$ erzielt. Die Wiederfindungsrate für reine Substanzen betrug $97 \%$ (Thiamin) bzw. 99\% (Thiaminpyrophosphat).

\section{A quantitative method for the simultaneous determination of thiamine and thiamine pyrophosphate in food or excreta}

A simple, specific, and rapid method for the estimation of thiamine and its pyrophosphoric ester is described. Hydrolysis of the phosphate groups is not necessary. After deproteinization, the sample is passed through a column of sephadex G-25 and determined by the thiochrome test. The best reproducibility was obtained in the range $0.5-3 \mu \mathrm{g}$. The recovery of pure substances was $97 \%$ for thiamine and $99 \%$ for cocarboxylase.

Die meisten Thiaminbestimmungen fußen auf der von JANSEN 1936 (1) eingeführten fluorometrischen Messung des Thiochroms, eines Oxydationsproduktes des Thiamins. Diese Messung hat den Vorteil, daß sie einfach ist und wenig Zeitaufwand beansprucht, hat aber den Nachteil, daß sie durch Nebenfluoreszenzen gestört wird. Man hat 2 Wege verfolgt, diese Nebenfluoreszenzen auszuschalten:

1. ihre Beseitigung aus der eigentlichen Analysenprobe,

2. ihre Kompensiefung durch eine Vergleichsprobe, in der die Thiochrombildung unterbunden oder die gebildete Thiochromfluoreszenz gelöscht wurde.

Für beide Richtungen lassen sich zahlreiche Autoren anführen (2).

Man hat versucht, das Thiamin durch Ausschütteln in saurer Lösung an Frankonit zu adsorbieren und den mit Thiamin angereicherten Frankonit direkt der Analyse zu unterwerfen (3-5). Auch die Extraktion der störenden Nebenfluoreszenzen aus dem angesäuerten $\mathrm{Ge}-$ misch mittels Isobutylalkohol vor der Oxydation des Thiamins $\mathrm{zu}$ Thiochrom wurde angewandt (6). Am erfolgreichsten war die Entfernung der Nebenfluoreszenzen aus dem Meßwert durch Säulenchromatographie an Permutit, Decalso, Dowex und Amberlite, sowie die Auftrennung der Vitamin $\mathrm{B}_{1}$-haltigen Substanzgemische durch die Papierchromatographie (siehe (2) S, 157/58 und (7)).

Von Anfang an wandte man sich auch dem zweiten Weg zu, der Kompensierung der Nebenfluoreszenzen durch eine Vergleichsprobe ohne Thiochrombildung. Diese Vergleichsprobe enthielt nur die Nebenfluoreszenzen, die vom Testwert subtrahiert wurden. Die Unterbindung der Thiochrombildung oder die Auslöschung der Thiochromfluoreszenzen geschah durch Weglassen des Oxydationsmittels, durch Zugabe von Natriumbisulfit vor der Oxydation, durch Blockierung der an der Thiochrombildung beteiligten Aminogruppen durch Benzolsulfochlorid oder durch pH-Verschiebung nach der Thiochrombildung ins extrem saure Gebiet (8). Allen aufgeführten Abänderungen der ursprünglichen Thiaminbestimmungsmethode von JANSEN ist gemeinsam, daß sie nur das Thiamin messen, weshalb vorher die Cocarboxylase mit Phosphatase zu Thiamin aufgespalten werden muß.

RoTH unternahm im Jahre 1938 (9) den Versuch, das Cocarboxylasethiochrom, das hydrophil ist und deshalb nicht mit Isobutanol, Butanol oder Amylalkohol extrahiert werden kann, in der wäßrigen Phase zu bestimmen. Diese Methode ist vermutlich noch anfälliger gegen Störfluoreszenzen als die Thiaminbestimmung. Sie wurde 1950 von Westendrink und SteYN-PARvé (10) als eine mögliche Methode zur Bestimmung des Thiaminpyrophosphats erwähnt und 1956 von OstrovskIJ (11), nach hohen Gaben von Thiamin an Versuchspersonen in kleinen Blutmengen angewandt. Als unterste Grenze der Bestimmbarkeit gibt er $1 \mathrm{mg}$ Cocarboxylase pro $1 \mathrm{~m} l$ Blut añ. Wegen der Unempfindlichkeit und Unsicherheit dieses Verfahrens haben wir eine neue Methode ausgearbeitet, die eine spezifische Bestimmung von Thiamin und seinem Pyrophosphatester erlaubt. Sie wird im folgenden mitgeteilt.

\section{Methodik}

\section{Prinzip der Methode}

Die hier dargestellte Methode erfaßt Thiamin und Thiaminpyrophosphat nebeneinander. Störfluoreszenzen schaltet man durch Auftrennung des zu untersuchenden Stoffgemisches an einer Sephadexsäule aus. Die beiden Formen des Vitamins werden oxydiert, nach Extraktion von Thiamin fluorimetrisch gemessen und die Ergebnisse mit Hilfe einer Eichkurve aus 3 oder 4 Testwerten reiner Substanzen ermittelt. 
Tab. 1

Bestimmung der Wiederfindungsrate nach Säulenpassage durch Vergleich mit der direkten Bestimmung Die Werte sind Durchschnittswerte aus je 3-4 Analysen. Sie geben die im Durchlauf gefundenen Mengen in Prozent der direkten Bestimmungen an. Die Streuungsprozente zeigen die mittlere quadratische Abweichung vọm Mittelwert an.

\begin{tabular}{cccccccc} 
& \multicolumn{2}{c}{ Thiamin } & \multicolumn{3}{c}{ Thiaminpyrophosphat } \\
$0,5 \mu \mathrm{g}$ & $1,0 \mu \mathrm{g}$ & $1,5 \mu \mathrm{g}$ & $2,0 \mu \mathrm{g}$ & $0,5 \mu \mathrm{g}$ & $1,0 \mu \mathrm{g}$ & $1,5 \mu \mathrm{g}$ & $2,0 \mu \mathrm{g}$ \\
\hline $98 \pm 1,6 \%$ & $99 \pm 1,7 \%$ & $98 \pm 1,3 \%$ & $99 \pm 0,9 \%$ & $100 \pm 1,9 \%$ & $99 \pm 2,6 \%$ & $98 \pm 1,2 \%$ & $102 \pm 0,9 \%$ \\
\hline
\end{tabular}

Erforderliche Reagenzien und Lösungen

Sephadex G-25 fine (Deutsche Pharmacia GmbH, Frankfurt) 0,1 proz. wäßr. $\mathrm{NaHSO}_{4}$-Lösung

fluoreszenzfreier Amylalkohol

15proz. $\mathrm{NaOH}$

2 proz. $\mathrm{K}_{3}\left[\mathrm{Fe}(\mathrm{CN})_{6}\right]$-Lösung

Oxydationsgemisch: $20 \mathrm{ml} 15$ proz. $\mathrm{NaOH}+3 \mathrm{~m} / 2$ proz. Kaliumhexacyanoferrat-Lösung.

Thiamin-Hydrochlorid, Cocarboxylase-Chlorid (Merck AG,

Darmstadt)

Apparate und Glasgeräte

Photometer mit Einrichtung zur Fluoreszenzmessung

Primärfilter $\mathrm{Hg} 313-366 \mathrm{~nm}$

Sekundärfiltè $400-3000 \mathrm{~nm}$

Fluoreszenzstandard 4709

Zubehör zum Eppendorfphotometer

Chromatographierohre von etwa $60 \mathrm{~cm}$ Länge und $10-11 \mathrm{~mm}$ Durchmesser (Büretten, Brand Wertheim, Qualität Goldbrand)

Schüttelzylinder mit Graduierung(Brand Wertheim, Qualität Goldbrand)

Glasküvetten $1 \mathrm{~cm}$ Schichtdicke

\section{Bescbreibung der Metbode}

Zur Auftrennung der Stoffgemische benutzen wir Sephadex G-25. $10 \mathrm{~g}$ trockenes Gel werden zum Quellen in soviel dest. Wasser suspendiert, daß nach der Sedimentation das überstehende Wasser um etwa 50\% höher im Glas steht als das gequollene Gel. Nach 3 Stdn ist die Quellung vollständig und das Gel kann benutzt werden. Wir füllen es in ein Chromatographierohr von etwa $10-11 \mathrm{~mm}$ Durchmesser und $60 \mathrm{~cm}$ Länge. Die so hergestellte Sephadexsäule kann mehrere Monate ohne Regeneration benutzt werden. Der zu untersuchende Extrakt wird mit 5 oder 10 proz. Trichloressigsäure enteiweißt und bei geringem Vitamin- $B_{1}-$ Gehalt im Vakuum-Rotationsverdampfer etwas eingeengt. Dann geben wir $0,2 \mathrm{~m} l$ der Probe, in denen $0,5-3 \mu \mathrm{g}$ Thiamin bzw. Thiaminpyrophosphat enthalten sein sollen, auf die Sephadexsäule. Man läßt einziehen und gibt noch $2 \mathrm{mal} 0,5 \mathrm{~m} l$ Elutionsmittel nach. Die auslaufenden Tropfen werden in einem graduierten Glaszylinder aufgefangen. Als Elutionsmittel dient 0,1 proz. Natriumhydrogensulfat. Die Tropfgeschwindigkeit beträgt etwa 6 Tropfen/Min. Bei der angegebenen Menge Sephadex und dem Durchmesser der Säule läßt man $33 \mathrm{ml}$ als Vorlauf auslaufen. Die nächsten $12 \mathrm{~m} /$ enthalten die gesamte Menge an Thiamin und Cocarboxylase. Diese $12 \mathrm{ml}$ werden gesondert in einem Meßzylinder aufgefangen und mit $1 \mathrm{ml}$ Oxydationsgemisch versetzt. Man läßt 2 Min. stehen und extrahiert dann das Thiamin durch 2 Min. langes Schütteln mit $13 \mathrm{ml}$ Amylalkohol. Thiaminpyrophosphat (Cocarboxylase) bleibt in der wäßr. Phase. Von beiden Phasen werden $5 \mathrm{ml}$ abgenommen, die amylalkoholische wird zur Klärung mit $0,5 \mathrm{~m} l 96$ proz. Äthylalkohol versetzt. Die Fluoreszenz von wäßr. und amylalkohol. Phase wird nacheinander im Eppendorfphotometer mit Fluoreszenzaufsatz gemessen. Wir benutzen das Primärfilter 313-366 und das Sekundärfilter 400-3000 nm. Als Bezugswert dient der Glasstandard 4709, der auf Anforderung von der Firma mitgeliefert wird. Dieser Standard wird in unserem Fall bei Schaltstufe 5 auf $100 \%$ Durchlässigkeit eingestellt. Nach Weiterschalten auf Stufe 7 werden dann die zu messenden Analysenwerte an der Durchlässigkeitsskala abgelesen und die entsprechenden Mengen an Thiamin und Cocarboxylase aus einer Eichkurve, deren Neigungswinkel von Tag zu Tag etwas schwankt, ermittelt. Die Eichkurve muß - für Thiamin und Thiaminpyrophosphat gesondert täglich neu aufgestellt werden.

\section{Ergebnisse}

Die Prüfung der Methode haben wir mit reinen Substanzen, Rattenleberextrakten, mit Suspensionen von Bäckerhefe, mit einem Extrakt aus Lactobacillus viridescens, mit Menschenharn, Apfelsinensaft und Weizenmehl durchgeführt. In einigen Fällen haben wir einen Vergleich mit der papierchromatographischen $\mathrm{Be}$ stimmung (7) vorgenommen und Zusätze reiner Substanzen zu Bäckerhefeextrakten und Apfelsinensaft untersucht.

\section{Bestimmung von reinen Substanzen nach Durchlauf durch die Sephadexsäulen}

Die Bestimmung von Gemischen reinen Thiamins und Thiaminpyrophosphats nach Sephadex-Behandlung wurde mit direkten Analysen derselben Substanzen ohne Säulendurchlauf verglichen (Tab. 1). Die Werte beider Gruppen stimmen nahezu überein.

\section{Untersuchungen von Rattenleberbomogenaten}

$25 \mathrm{~m} l$ Homogenat (Potter-Elvehjem-Methode) einer ganzen Rattenleber in dest. Wasser, das mit Essigsäure auf $\mathrm{pH}$ 3,5 eingestellt worden war, wurden mit $25 \mathrm{ml}$ 5proz. Trichloressigsäure versetzt, $20 \mathrm{Min}$. bei $8000 \mathrm{U}$./ Min. zentrifugiert, einmal mit 5proz. Trichloressigsäure nachgewaschen und wieder zentrifugiert. Die vereinigten Überstände wurden vorsichtig mit 15 proz. $\mathrm{NaOH}$ auf pH 3,5 eingestellt, auf etwa $4 \mathrm{ml}$ eingeengt, auf $5 \mathrm{ml}$ aufgefüllt und $45 \mathrm{Min}$. bei $12000 \mathrm{U}$./Min. zentrifugiert. Der Überstand wurde im Kühlschrank aufbewahrt. Doppelanalysen an 2 Säulen ergaben die Werte der Tabelle 2.

Tab. 2

Thiamin- und Thiaminpyrophosphatgehalt von Rattenleber. Doppelwerte der Analysen von 2 verschiedenen Säulen I u. II.

\begin{tabular}{|c|c|c|c|c|c|c|}
\hline \multirow[t]{2}{*}{$\begin{array}{l}\text { Präparat } \\
\text { Nr. }\end{array}$} & \multirow[t]{2}{*}{$\begin{array}{c}\text { Präparation } \\
\text { am }\end{array}$} & \multirow[t]{2}{*}{$\begin{array}{c}\text { Analyse } \\
\text { am }\end{array}$} & \multicolumn{2}{|c|}{$\underset{(\mu \mathrm{g} / \text { Leber) }}{\text { Thiamin }}$} & \multicolumn{2}{|c|}{$\begin{array}{l}\text { Thiaminpyrophos } \\
\text { phat ( } \mu \mathrm{g} / \mathrm{Leber})\end{array}$} \\
\hline & & & I & II & I & II \\
\hline 1 & 17. 9.68 & 19. 9.68 & 6,94 & 7,20 & 85,8 & 89,9 \\
\hline 2 & 17.9 .68 & 20.9 .68 & 3,85 & 3,85 & 102,5 & 102,8 \\
\hline 3 & 23. 9.68 & 25.9 .68 & 7,10 & 7,32 & 103,3 & 104,4 \\
\hline
\end{tabular}

\section{Untersucbung von Bäckerbefe}

7,5 g Bäckerhefe wurden in wenig auf $\mathrm{pH}$ 3,5 angesäuertem Wasser mit Seesand gemörsert und mit $30 \mathrm{ml} 5$ proz. Trichloressigsäure enteiweißt. Nach $15 \mathrm{Min}$. Stehen wurde $15 \mathrm{Min}$. bei $4000 \mathrm{U}$./Min. zentrifugiert, der Rückstand einmal mit 5proz. Trichloressigsäure nachgewaschen und die vereinigten Uberstände auf etwa $2,5 \mathrm{ml}$ eingeengt, nachdem vorher mit 15 proz. $\mathrm{NaOH}$ 
auf $\mathrm{pH} 3,5$ eingestellt worden war. Die $2,5 \mathrm{~m} l$ wurden übergespült und auf $5 \mathrm{ml}$ mit dest. Wasser, das mit Essigsäure auf $\mathrm{pH} 3,5$ gebracht worden war, aufgefüllt. Danach wurde etwa $40 \mathrm{Min}$. bei 12000 U./Min. zentrifugiert und der Überstand im Kühlschrank aufbewahrt. Die Ergebnisse der Analysen zeigt Tabelle 3.

\section{Untersucbung von Lactobacillus viridescens}

Verwendet wurde eine Kultur von Lactobacillus viridescens, die 12 Stdn. ohne Substrat und anschließend $5 \mathrm{Min}$. in einer Nährlösung mit Zusatz von $100 \mu \mathrm{g}$ Thiaminchloridhydrochlorid gehalten wor den war. Nach Verreiben mit Quarzsand und Aufnahme in zentrifugiert. Der Uberstand wurde auf kleine Fläschchen verteilt und bei $-20^{\circ}$ in der Kühltruhe aufbewahrt. Aus jeder Probe wurden Doppelanalysen von je $0,2 \mathrm{~m} l$ auf je 2 Säulen gemacht. Das Ergebnis zeigt Tabelle 4.

\section{Analysen von Menschenbarn}

$100 \mathrm{ml}$ Harn wurden mit Essigsäure auf $\mathrm{pH} 3,5$ gebracht und bis auf etwa $15 \mathrm{~m} /$ eingeengt, übergespült und auf Trispuffer von $\mathrm{pH} \mathrm{6,5}$ wurde mit hoher Tourenzahl

$20 \mathrm{~m} l$ mit dest. Wasser aufgefüllt. Es wurden verschiedene Mengen auf die Säulen gegeben.

Thiaminpyrophosphat ist im Normalharn nicht enthalten, nach Gabe großer Dosen Vitamin $B_{1}$ werden in der wäßrigen Phase jedoch Thiaminderivate gefunden, worauf wir in der Diskussion zurückkommen werden.

\section{Untersucbung von Apfelsinensaft}

Der Saft einer frischen Apfelsine wurde bei $40^{\circ}$ Badtemperatur auf $10 \mathrm{ml}$ eingeengt, dann zentrifugiert (30 Min. bei $12000 \mathrm{U}$./Min.) und je $0,2 \mathrm{ml}$ auf 2 Säulen gegeben. Das Ergebnis zeigt Tabelle 6. Enteiweißung war nicht erforderlich.

\section{Untersuchung von Weiqenauszugsmehl, Type 405 obne Vita- minzusatz}

$5 \mathrm{~g}$ Mehl wurden in $25 \mathrm{ml}$ dest. Wasser eingerührt, $25 \mathrm{ml}$ 10proz. Trichloressigsäure zur Eiweißfällung zugegeben, zentrifugiert (15 Min. bei $5000 \mathrm{U}$./Min.), der Úberstand abgegossen, mit $\mathrm{NaOH}$ auf $\mathrm{pH} 3,5$ gebracht und bei $40^{\circ}$ Badtemperatur bis auf $5 \mathrm{ml}$ eingeengt, wieder zentrifugiert und je $0,2 \mathrm{ml}$ auf 2 Säulen gegeben. Das Ergebnis zeigt Tabelle 7.

Thiamin- und Thiaminpyrophosphatgehalt von Hefe. Doppelanalysen an 2 Sephadexsäulen I und II

\begin{tabular}{|c|c|c|c|c|c|c|}
\hline \multirow[t]{2}{*}{$\begin{array}{l}\text { Präparation } \\
\text { am }\end{array}$} & \multirow[t]{2}{*}{$\begin{array}{c}\text { Analyse } \\
\text { am }\end{array}$} & \multicolumn{2}{|c|}{$\begin{array}{c}\text { Thiamin } \\
(\mu \mathrm{g} / 100 \mathrm{~g} \\
\text { Hefe })\end{array}$} & \multicolumn{2}{|c|}{$\begin{array}{c}\text { Thiaminpyro- } \\
\text { phosphat } \\
(\mu \mathrm{g} / 100 \mathrm{~g} \text { Hefe) }\end{array}$} & \multirow[t]{2}{*}{$\begin{array}{c}\text { Gesamt- } \\
\text { Thiamin } \\
\text { ( } \mu \text { g/100 } \\
\text { Hefe) }\end{array}$} \\
\hline & & 1 & II & I & II & \\
\hline 30.9 .68 & $\begin{array}{l}1.10 .68 \\
2.10 .68 \\
3.10 .68 \\
4.10 .68\end{array}$ & $\begin{array}{l}44,8 \\
38,7 \\
35,3 \\
75,0\end{array}$ & $\begin{array}{l}44,8 \\
37,3 \\
35,3 \\
77,8 *\end{array}$ & $\begin{array}{r}964 \\
954 \\
1010 \\
930\end{array}$ & $\begin{array}{c}953 \\
934 \\
1013 \\
935^{*}\end{array}$ & $\begin{array}{l}742 \\
724 \\
770 \\
752\end{array}$ \\
\hline
\end{tabular}

* Wahrscheinlich ist ein Teil der Cocarboxylase zu Thiamin abgebaut worden.
Tab. 4

Thiamin- und Thiaminpyrophosphatgehalt von Lactobacillus viridescens.

\begin{tabular}{|c|c|c|c|c|c|}
\hline \multirow[t]{2}{*}{$\begin{array}{c}\text { Präparation } \\
\text { am }\end{array}$} & \multirow[t]{2}{*}{$\begin{array}{l}\text { Analyse } \\
\text { am }\end{array}$} & \multicolumn{2}{|c|}{$\begin{array}{c}\text { Thiamin } \\
(\mu \mathrm{g} / \mathrm{ml} \text { Uberstand) }\end{array}$} & \multicolumn{2}{|c|}{$\begin{array}{l}\text { Thiaminpyrophospha } \\
\text { ( } \mu \mathrm{g} / \mathrm{m} l \text { Ưberstand })\end{array}$} \\
\hline & & 1 & II & I & II \\
\hline 20. 3. 69 & $\begin{array}{r}24.3 .69 \\
25.3 .69 \\
31.3 .69 \\
1.4 .69 \\
2.4 .69\end{array}$ & $\begin{array}{r}10,20 \\
9,49 \\
9,85 \\
10,12 \\
10,03\end{array}$ & $\begin{array}{r}10,24 \\
9,67 \\
10,42 \\
10,28 \\
10,03\end{array}$ & $\begin{array}{r}9,58 \\
9,57 \\
9,69 \\
9,97 \\
10,28\end{array}$ & $\begin{array}{r}9,56 \\
9,79 \\
9,97 \\
10,08 \\
10,25\end{array}$ \\
\hline
\end{tabular}

Tab. 5

Thiamingehalt von Menschenharn bei unterschiedlicher Aufnahme von Vitamin-B.. Der Harn wurde anschließend an die Periode der Vitamin$B_{1}$-Einnahme asserviert

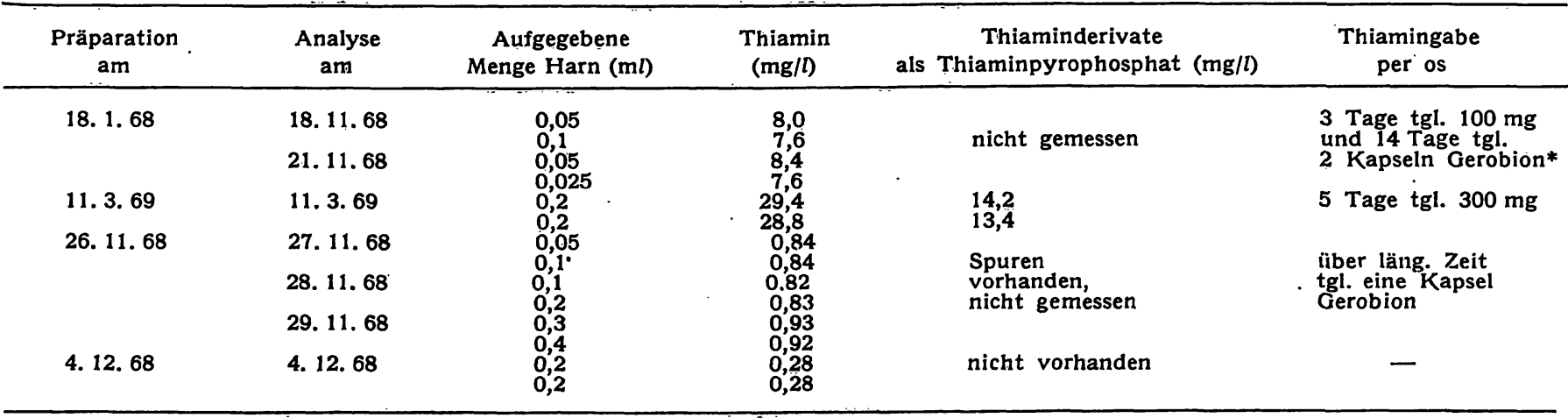

*) Eine Kapsel Gerobion enthält nach Angabe des Herstellers 4 mg Cocarboxylase.

Tab. 6

Thiamin- und Thiaminpyrophosphatgehalt von Apfelsinensaft

\begin{tabular}{|c|c|c|c|c|}
\hline \multirow{2}{*}{ Analyse am } & \multicolumn{2}{|c|}{ Thiamin $(\mu \mathrm{g} / 100 \mathrm{ml})$} & \multicolumn{2}{|c|}{$\begin{array}{c}\text { Thiaminpyrophosphat } \\
(\mu \mathrm{g} / 100 \mathrm{ml})\end{array}$} \\
\hline & I & II & I & II \\
\hline $\begin{array}{l}6.12 .68 \\
28.2 .69\end{array}$ & $\begin{array}{l}61,6 \\
61,6\end{array}$ & $\begin{array}{l}61,7 \\
67,3\end{array}$ & $\begin{array}{l}76,0 \\
83,2\end{array}$ & $\begin{array}{l}66,6 \\
79,9\end{array}$ \\
\hline
\end{tabular}

Tab. 7

Thiamin- und Thiaminpyrophosphatgehalt von Weizenmehl Type 405

\begin{tabular}{|c|c|c|c|c|}
\hline \multirow{2}{*}{ Analyse am } & \multicolumn{2}{|c|}{ Thiamin $(\mu \mathrm{g} / 100 \mathrm{~g})$} & \multicolumn{2}{|c|}{$\begin{array}{c}\text { Thiaminpyrophosphat } \\
(\mu \mathrm{g} / 100 \mathrm{~g})\end{array}$} \\
\hline & I & II & I & II \\
\hline 12. 12.68 & 23,3 & 21,2 & - & - \\
\hline
\end{tabular}




\section{Diskussion}

Die Beispiele ergeben, daß die Methode allseitig zu verwenden ist, sobald mindestens $0,1 \mu \mathrm{g}$ Thiamin bzw. -pyrophosphat in der Probe, die auf die Säule gegeben wird, enthalten ist. Die günstigste Menge liegt zwischen 0,5 und $3 \mu \mathrm{g}$. Es war nicht beabsichtigt, vollständig ausgearbeitete Methoden zur Aufarbeitung der verschiedenen Produkte zu geben. Dagegen wurden in einigen Fällen Zusätze von reinen Substanzen getestet in allen Fällen wurde die Probe so gewählt, daß die in ihr enthaltene Thiamin- bzw. Thiaminpyrophosphatmenge in etwa den zugesetzten Mengen entsprach (Tab. 8) - und die Ergebnisse dieser Methode mit
Tab. 8

Wiederfindungsrate für $\mathrm{zu}$ analysierten Proben zugesetztes Thiamin bzw. Thiaminpyrophosphat

\begin{tabular}{|c|c|c|c|c|c|}
\hline \multirow{3}{*}{ Analyse } & \multirow{3}{*}{ Probe } & \multicolumn{2}{|c|}{ Zusatź' } & \multicolumn{2}{|c|}{ Wiedergéfunden } \\
\hline & & \multicolumn{4}{|c|}{$\begin{array}{cc}\text { Thiamin- } & \text { Thiamin- } \\
\text { Thiamin pyrophosphat Thiamin pyrophosphat }\end{array}$} \\
\hline & & $(\mu \mathrm{g})$ & $(\mu \mathrm{g})$ & $(\%)$ & $(\%)$ \\
\hline *24./28. 4. & Bäckerhefe & 0,4 & 1,0 & 92 & 96 \\
\hline 29./30. 4 . & Bäckerhefe & 0,75 & 1,0 & 105 & 101 \\
\hline 6.5. & Apfelsine & 1,67 & 1,67 & 96 & 98 \\
\hline 6.5 . & Apfelsine & 1,67 & 1,67 & 95 & 99 \\
\hline
\end{tabular}

*) Da die Bäckerhefe $10 \mathrm{mal}$ soviel Cocarboxylase wie Thiamin enthält, konnten die beiden Zusätze nicht zur gleichen Verdünnung zugegeben werden.

Tab. 9

Vergleich der hier angegebenen Methode mit der papierchromatographischen Bestimmung (7)

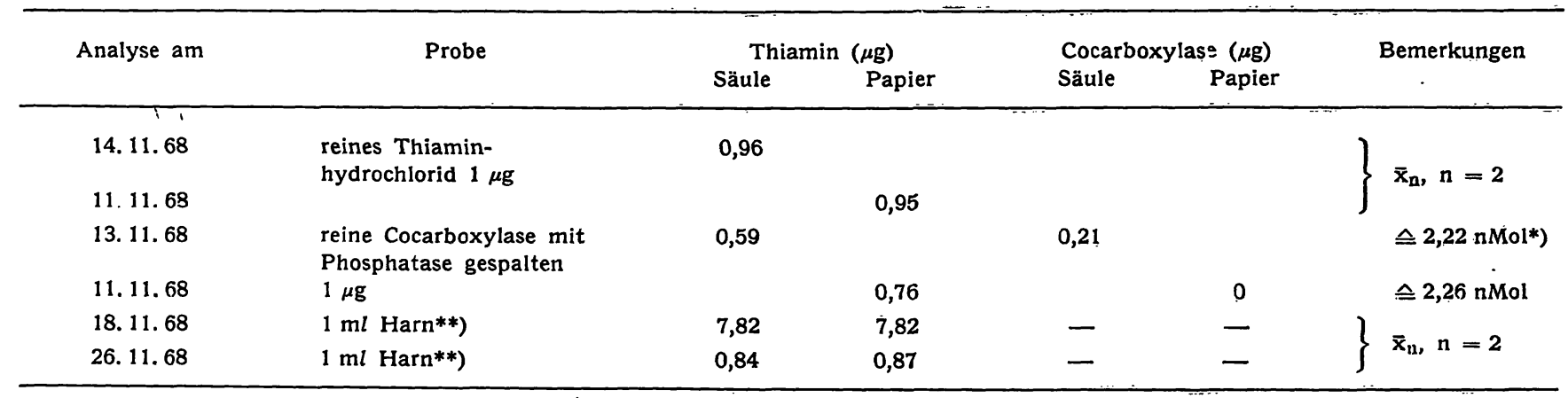

*) Im Falle der Papierchromatographie wurde die Cocarboxylase quantitativ durch Phosphatase in Thiamin umgewandelt. Bei der Aufarbeitung für die Säulen war die Aufspaltung nicht vollständig. (2 S76) Es wurden deshalb die gefundenen nMol miteinander verglichen.

denen der papierchromatographischen Bestimmung (7) verglichen (Tab. 9).

Wie schon erwähnt, wird im Normalharn kein Thiaminpyrophosphat gefunden. Beim Ausschütteln des Säuleneluats mit Amylalkohol ohne vorhergehende Oxydation von Harn nach Gabe hoher Dosen - die Versuchsperson hatte vor dem Sammeln des Harns 14 Tage lang 2 Kapseln Gerobion und 3 Tage $100 \mathrm{mg}$ Thiamin täglich eingenommen - zeigte sich in der alkoholischen Phase eine leichte Blaufluoreszenz. Sie stammt wahrscheinlich von ausgeschiedenem Thiochrom: bei Ansäuern verschwindet sie und tritt nach Alkalischmachen wieder auf; ein für Thiochrom typisches Verhalten.

Nach Oxydation im Alkalischen war die alkoholische Phase bei Ausschütteln des Säuleneluats mit Amylalkohol stark blau. Die wäßrige Phase fluoreszierte ebenfalls blau.

Um festzustellen, welche Substanzen ausgeschieden werden, wurden die einzelnen Phasen auf Chromatographiepapier getropft. Die einfache amylalkoholische Ausschüttelung, ohne vorhergehende Oxydation, zeigt keine Fluoreszenz. Die Menge war also trotz Einengens zu gering. Die alkoholische Phase nach Oxydation, zeigte viel Thiamin, aber keine andere Substanz. Die wäßrige Phase des alkoholischen Auszugs nach Oxydation zeigte auf dem Papier zwei Flecke, wahrscheinlich 2 Phosphatester des Thiamins. Die Natur dieser Substratflecke haben wir aber nicht näher untersucht.
Tab. 10

Mittlere quadratische Abweichung bei der Bestimmung verschiedener Mengen von Thiamin bzw. Thiaminpyrophosphat

\begin{tabular}{ccccccc}
\hline $\begin{array}{c}\text { Thiamin } \\
(\mu \mathrm{g})\end{array}$ & $\mathrm{n}$ & $\mathrm{s}^{2}(\%)$ & Thiaminpyrophosphat & $\mathrm{n}$ & $\mathrm{s}^{2}(\%)$ \\
\hline 0,5 & 17 & 1,7 & 0,5 & 17 & 4,1 \\
1,0 & 17 & 3,6 & 1,0 & 17 & 7,1 \\
1,5 & 17 & 1,6 & 1,5 & 17 & 3,3 \\
2,0 & 17 & 2,3 & 2,0 & 17 & 3,2 \\
\hline
\end{tabular}

Als Letztes errechneten wir aus je 17 Analysen die mittlere quadratische Abweichung (Tab. 10), die für Thiamin bei der Berechnung aller $x_{1}$-Werte $\pm 2,3 \%$, für Thiaminpyrophosphat $\pm 4,6 \%$ ergab. Die zugrunde liegenden Analysen erstreckten sich über einen Zeitraum von einem Monat, enthalten also auch die täglichen Schwankungen der Eichkurven und des Leerwertes.

Stellt man aus den einfächen Mittelwerten jeder Reihe von 17 Analysen, die Eichreihen für Thiamin und Thiaminpyrophosphat auf, so ergeben sich lineare Eichkurven: für Thiamin mit dem errechnetèn Leerwert von $L=16,2$ Skalenteilen und dem Eichkurvenfaktor $\mathrm{F}=0,041$, für Cocarboxylase $\mathrm{L}=13,6$ Skalenteile und $F=0,048$. Berechnet man die abgelesenen Durchlässigkeitsprozente $\operatorname{der} \mathrm{zu}$ bestimmenden Analysenproben mit diesen Leer- und Faktorwerten, so tritt für Thiamin eine mittlere quadratische Abweichung von $\pm 2,3 \%$ und für Cocarboxylase von $\pm 6,2 \%$, aus 52 Werten ermittelt, auf. Es ist also eine schnelle Orien- 
tierung möglich, wenn man etwas geringere Anforderungen an die Genauigkeit der Analysenergebnisse stellt.

Der große Vorteil der Methode liegt darin, daß sie wenig Zeit und Aufwand erfordert, daß man auf die Aufspaltung von Thiaminpyrophosphat zu Thiamin und auf möglichst vollständige Enteiweißung verzichten kann, und daß die Regeneration der Säulen, die sehr viel Zeit beansprucht, entfällt. Es muß allerdings bemerkt werden, daß man, wie bei den einfachen Analysen mit Aufspaltung von Thiaminpyrophosphat zu Thiamin, in der wäßrigen Phase alle Phosphatester zusammen erfaßt.

Ich danke Fräulein SCHLIEKER für ihre zuverlässige Mitarbeit.

\section{Literatur}

1. Jansen, B. C. P., Recueil Trav. chim. Pays-Bas 55, 1046 (1936) 2. Gstirner, F., Chem.-phys. Vit.-Best. Meth., Seite 68-111, Enke-Verlag Stuttgart (1965). - 3. Karrer, P., Helv. chim. Acta 20, 1147 (1937). - 4. Flavier, H. und L. Genevors, C. R. Séances Soc. Biol. Filiales 130, 497 (1939). - 5. WestenbrinK, H. und J. Goudsmit, Ned. tschr. geneesk. 81, 2632 (1937). 6. Ritsert, K., Klin. Wschr. 17 (1938). - 7. KRAUT, H. und
L. WiLdeManN, Internat. Zschr. Vitaminforsch. Bern 27, 122 (1956); Kaiser, H. und L. WildemanN, ebenda 27, 131 (1956). 8. KNовцосн, E., Phys.-Chem. Vit.-Best. Meth., Seite 174, Akademie-Verlag Berlin (1963). - 9. RотH, H., Biochem. Z. 297, 52 (1938). - 10. Westendrink, H. G. K. und E. P. Steyn-Parvé, Internat. Zschr. Vitaminforsch. Bern 21, 461 (1950). - 11. OsTRovskrJ, Yu. M., Bjull. eksper. biol. med. Moskva 42, 72 (1956).
Dr. Liesel Wildemann

Max-Planck-Institut für Ernährungsphysiologie 4600 Dortmund, Rheinlanddamm 201 